\title{
$25^{\text {th }}$ International Conference \\ ENGINEERING MECHANICS 2019
}

Svratka, Czech Republic, 13 - 16 May 2019

\section{ELECTROCHEMICAL MACHINING OF CONICAL SURFACES}

\begin{abstract}
J. Sawicki*
Keywords: The paper presents mathematical modelling of contactless ECM of conical surfaces. Applying the known-from-literature equation describing the evolution of the machined surface shape and the flow equation for the mixture or electrolyte and hydrogen in the interelectrode gap, there were determined parameters characteristic for ECM. For the machining parameters set, the calculations results for selected ECM parameters are shown in diagrams.
\end{abstract}

Keywords: electrochemical machining, electrolyte flow, computer simulation

\section{Introduction}

Unconventional manufacturing methods have been widely applied in aviation, automotive, arms, electronics and household appliances sectors, especially to manufacture elements from difficult-to-cut materials (Kozak, 1976 and Davydov et al., 2004).

Unconventional manufacturing methods include electrochemical contactless machining which, for many years, has been intensively developed both at home and abroad. Introducing new alloys, composite materials in numerous devices, vehicles and tools very frequently requires the application of unconventional machining methods (Dąbrowski et al., 2005).

Electrochemical machining covers a group of machining methods which involve electrochemical digestion. Of various ECM variations, electrochemical machining is a variation of contactless machining most often used to manufacture parts of machinery and tools (Ruszaj, 1998). Electrochemical machining involves anode digestion of metal layers (of the workpiece) removed together with electrolyte from the interelectrode gap. In the interelectrode gap a mixture of electrolyte, hydrogen atoms and electrochemical digestion products is created (Paczkowski et al., 2008).

The physical phenomena which occur in the interelectrode gap determine the accuracy of electrochemical machining operations. To mathematically describe the ECM process, the equation of the evolution of the workpiece shape and the equation of electrolyte movement in the interelectrode gap: the continuity of flow, momentum and energy are used (Sawicki, 2013).

The essence of ECM computer modelling is to determine the evolution of the shape of the surface machined, namely the anode.

The aim of the paper has been the mathematical analysis of electrochemical machining of conical surfaces facilitating the determination of the shape of conical surface after machining within the set time.

\section{Mathematical electrochemical machining process modelling}

*Assoc. Prof. Jerzy Sawicki: UTP University of Science and Technology, Bydgoszcz, Faculty of Mechanical Engineering, jerzy.sawicki@utp.edu.pl. 
The flow area of the mixture of electrolyte and hydrogen in the interelectrode gap (IEG) is presented in figure 1.

The tool electrode surface (TE) is described with function $R(x)$ which is a radius of its area. The thickness of interelectrode gap (IEG) $h$ stands for the section defined along normal to the surface of the tool electrode.

Gap thickness $\mathrm{h}(\mathrm{x})$ is low, as compared with the radius of the electrode surface $R(x)$. Hence:

$$
h(x)<R(x)
$$

The equation of the evolution of the shape of machined, surface due to anode digestion, assumes the form of (Kozak, 1976):

$$
\frac{\partial Z_{A}}{\partial t}=k_{V} j_{A} \sqrt{1+\left(\frac{\partial Z_{A}}{\partial R}\right)^{2}}
$$

for $t=0 \quad Z_{A}=Z_{A}(R)$.

Assuming that the potential distribution along the normal section to the tool electrode is linear (often assumed in technological calculations), the current density function

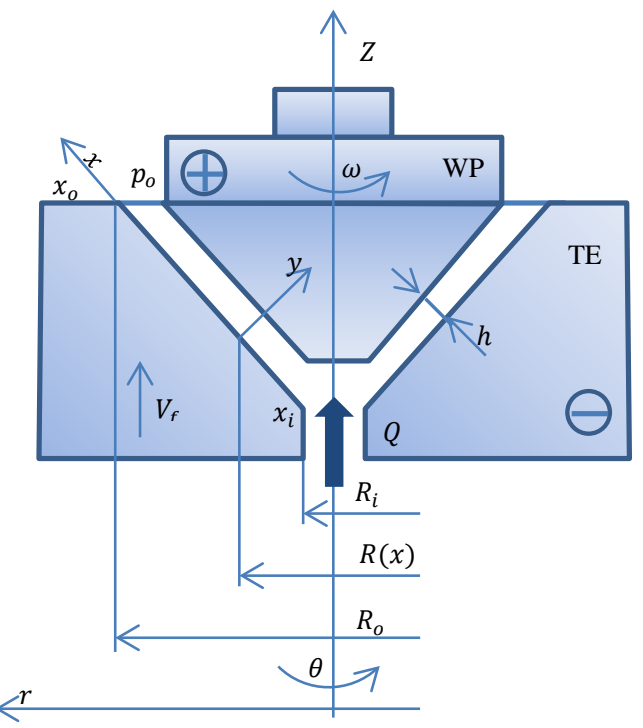

Fig. 1. Area of the flow of electrolyte in the gap (WP - workpiece, TE- tool electrode) can be expressed as (Sawicki, 2013):

$$
j_{A}=\kappa_{0} \Phi_{\mathrm{TG}}^{-1} \frac{U-E}{h}
$$

here: $h$-the lowest interelectrode thickness, $U$ - working voltage between electrodes, $E$ - summary electrode polarisation.

The lowest interelectrode gap thickness is the distance between the point on the workpiece (anode) and the point on the surface of the tool electrode (TE) described with the following dependence:

$$
Z_{e}(R, t)=f\left(R_{e}\right)+v_{f} t
$$

where: $v_{f}$ - velocity of the feed motion of the tool electrode (TE),

Function $\Phi_{\mathrm{TG}}$ describes changes in conductivity in the interelectrode gap (IEG) triggered by variable temperature field and the volumetric concentration of the gas phase and it is determined as follows:

$$
\Phi_{\mathrm{TG}}=\frac{1}{h}\left[\int_{0}^{h} \frac{d y}{\left(1+\alpha_{T} \Delta T\right)(1-\beta)^{\frac{3}{2}}}\right]
$$

where: $\alpha_{T}$ - temperature coefficient of electrical conductivity, $\Delta T$ - increase in mixture temperature.

To determine the evolution of the workpiece shape (solution to the equation (2), it is necessary to determine the temperature increases $\Delta T=T$-To and the gas-phase concentration distribution $\beta$. It requires solving the set of equations for electrolyte movement in the interelectrode gap (IG).

The mathematical modelling of electrolyte flow in the interelectrode gap was performed in a locally orthogonal coordinate system related to the electrode $x, y$ (Sawicki, 2013) .

Treating the electrolyte as a mixture of it electrolyte and hydrogen, equations of motion resulting from the following principles: conservation of mass, momentum and energy in a locally orthogonal coordinate system, assuming a laminar flow assume the following form (Sawicki,2013):

- equations of flow continuity for electrolyte and hydrogen, respective

$$
\frac{1}{R} \frac{\partial\left(\rho_{e} R v_{x}\right)}{\partial x}+\frac{\partial\left(\rho_{e} v_{y}\right)}{\partial y}=0, \frac{1}{R} \frac{\partial\left(\rho_{h} R v_{x}\right)}{\partial x}+\frac{\partial\left(\rho_{h} v_{y}\right)}{\partial y}=j \eta_{h} k_{h} h^{-1}
$$

- momentum equation:

$$
\rho_{e} v_{\theta}^{2} \frac{R^{\prime}}{R}=-\frac{\partial p}{\partial x}+\frac{\partial}{\partial y} \mu\left(\frac{\partial v_{x}}{\partial y}\right), \quad 0=\frac{\partial}{\partial y} \mu\left(\frac{\partial v_{\theta}}{\partial y}\right) \quad 0=-\frac{\partial p}{\partial y}
$$

here: $v_{x}, v_{\theta}, v_{y}$ - components of velocity vector, $p$ - pressure of the mixture of electrolyte and hydrogen, $\rho_{e}=(1-\beta) \rho_{e}^{0}$ - equivalent electrolyte density, $\rho_{h}=\beta \rho_{h}^{0}$ - equivalent hydrogen density, $\mu$ - dynamic coefficient of electrolyte viscosity.

Disregarding the dissipation of mechanical energy, considering the Joule heat released due to the flow of electric current, forced heat convection caused by electrolyte flow, heat exchange by electrode, the regression equation assumes the following form (Kozak, 1976, Sawicki, 2013): 


$$
v_{x} \frac{\partial T}{\partial x}+v_{y} \frac{\partial T}{\partial y}=\frac{\partial}{\partial y}\left(a \frac{\partial T}{\partial y}\right)+\frac{j_{A}^{2}}{\rho_{e} c_{p} \kappa}
$$

here: $T$ - temperature, $a$ - thermal diffusivity of electrolyte, $\kappa$ - electrical specific conductance of electrolyte, $c_{p}$ - specific heat at constant pressure.

The system of equations (6)-(8) formulated is the basic system of equations of the flow for the analysis of axisymmetric flow of the mixture of electrolyte and hydrogen in the interelectrode gap.

For the gap supplied with electrolyte with constant value of volumetric flow rate $Q=$ const., disregarding the local losses on the inlet and outlet, the solution to equations (6)-(8) should meet the boundary conditions:

- velosity components:

$$
v_{x}=v_{y}=0 \text { gdy } y=0, v_{x}=0, v_{y}=0 \text { gdy } y=h, v_{\theta}=0 \text { gdy } y=0, v_{\theta}=\omega R(x) \text { gdy } y=h
$$

- pressure: $p=p_{o}$ dla $x=x_{o}$

- for temperature: on the walls: $T=T_{s}$ for $x \geq x_{i}$ and $y=0$ and $y=h$, on the inlet, namely when $x=x_{i}, T=T_{i}$

here: $p_{o}$ - pressure on the interelectrode gap outlet, $x_{i}, x_{o}$ - coordinates of the location of the gap inlet and outlet, $T_{s}$ - temperature of electrodes, $T_{i}$ - temperature of electrolyte on the inlet.

System of equations (6) - (8) was analytically solved by defining the distributions of velocity and pressure in the interelectrode gap (IEG) with a constant thickness. The value of the thickness of the gap of a given cross-section is numerically determined by solving the equation of evolution at a given time step. Solving the energy equation (8) with the finite difference method, the temperature distribution across and along the gap was determined. The knowledge of physical fields of the flow of electrolyte facilitated an effective determination of the shape of the workpiece (anode). The computer algorithm of the electrochemical shape surface machining simulation is presented in paper (Sawicki, 2013).

\section{Computer simulation results}

The calculations were performed for the set machining time, assuming the passivating electrolyte. The calculations assumed the following essential machining parameters:

Table.1. Machining parameters

\begin{tabular}{lccclccc}
\hline Initial gap & $h$ & $\mathrm{~mm}$ & 0.2 & Volumetric flow rate & $Q$ & $\mathrm{dm}^{3} \min ^{-1}$ & 3 \\
Feed motion velocity (TE) & $V_{f}$ & $\mathrm{~mm} \mathrm{~s}^{-1}$ & 0.1 & Pressure & $p_{o}$ & $\mathrm{MPa}$ & 0,1 \\
Interelectrode voltage & $U$ & $\mathrm{~V}$ & 15 & Machining time & $t$ & $\mathrm{~s}$ & 60 \\
\hline
\end{tabular}

The calculations results are graphically presented in Figs 2-7.

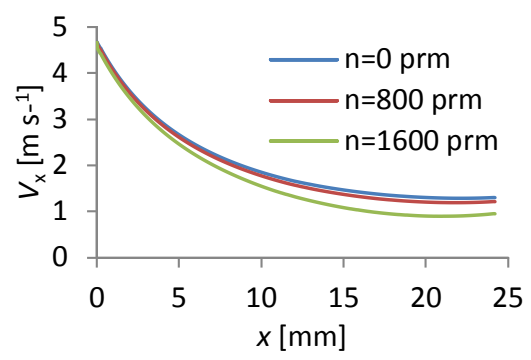

Fig. 2. Electrolyte flow velocity $v_{x}$

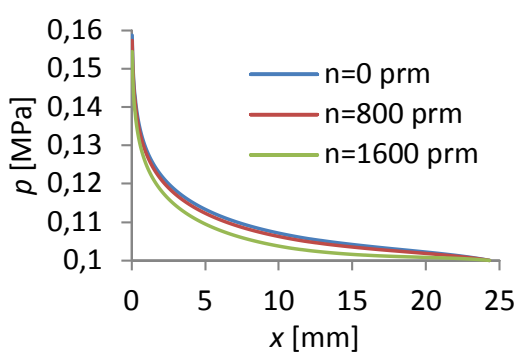

Fig. 3. Pressure $p$

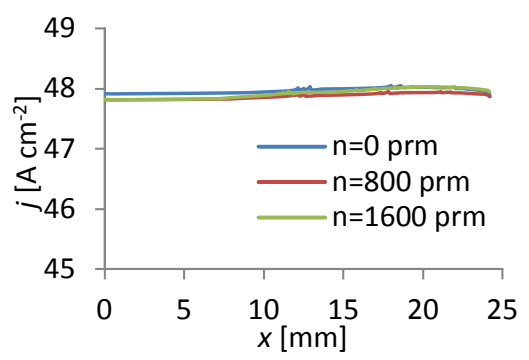

Fig. 4. Current density $j$ 


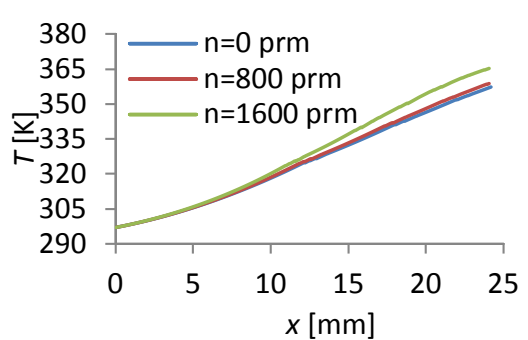

Fig. 5. Temperature T
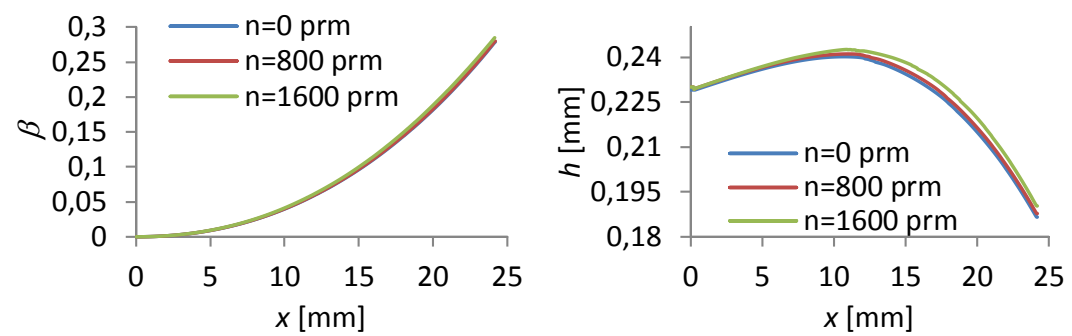

Fig. 6. Volumetric concentration of hydrogen $\beta$ Fig. 7. Gap height IEG $h$,

\section{Conclusions}

The paper presents mathematical ECM conical rotary surfaces modelling. Using electrochemical shaping known from theory, the equation of evolution of the machined surface shape and the equations of flow of the mixture of electrolyte and hydrogen resulting from the balance of mass, moment and energy, after introducing adequate simplifications, the problem was partially analytically and numerically solved, obtaining the distributions of the gap stiffness, current density, volumetric concentration of gas phase, temperature, pressure and velocity along the interelectrode gap.

The computer simulation facilitated obtaining information on the course of machining, especially in reference to the physical conditions in the interelectrode gap (IEG). The results are illustrated in figures (Fig.2-7).

The distributions of ECM parameters presented in Fig. 3 show that the application of the rotational motion of one of the electrodes has a significant effect on machining conditions. An increase in rotational velocity of the workpiece (WP) results in:

- a decrease in velocity $v_{x}$ along the gap (IEG), especially at the outlet from the gap, which is related to the effect on the values of that velocity, peripherial velocity of electrolyte flow $v_{\theta}$ caused by rotating movement of the workpiece (WP),

- a decrease in pressure $p$ along the gap (IG), which can, at considerable rotational speeds and excessive flow rates of electrolyte $Q$, lead to dangerous negative pressures, in extreme cases allowing for the emergence of critical conditions,

- an increase in current density $j$, which is a result of both a decrease in electrolyte flow velocity and a decrease in pressure.

- an increase in current density $j$ is clearly visible close to the gap outlet (IEG), an increase in mean temperature at the gap outlet (IEG), which results from an increase in current density $j$ in that part of the gap (IEG)

- inconsiderable increase in volumetric hydrogen concentration $\beta$, accompanied by an increase in current density results in an increase in gap thickness IEG), which means a better electrochemical digestion of the workpiece (WP).

The ECM modelling method facilitates a computer analysis of the so-called inverse problem, namely the problem aiming at obtaining the right shape of the tool electrode (TE) for the set tolerance of the workmanship of the workpiece (WP). Inverse problem involves a comparison of the results of simulation of the evolution of the workpiece shape from i- iteration with the excepted final workpiece (WP) shape.

\section{References}

Davydov A.D., Volgin V.M., Lyubimov V.V., Electrochemical Machining of Metals: Fundamentals of Electrochemical Shaping, Russian Journal of Electrochemistry, Vol. 40, (12), pp. 1230-1265, 2004,

Dąbrowski L., Paczkowski T., Computer Simulation of Two-dimensional Electrolyte F1ow in Electrochemical Machining. Russian Journal of Electrochemistry 41(1), pp. 91-98, 2005,

Kozak J., Mathematical Models for Computer Simulation of Electrochemical Machining Process. Journal of Materials Processing Technology, Vol. 76, 1976,

Paczkowski T., Sawicki J., Electrochemical Machining of Curvilinear Surfaces, Journal: LMST: Machining Science and Technology 12 (1), Philadelphia, United States, pp.33-52, 2008,

Ruszaj A., Application of ECM Machining in the Polish Industry. Mat. SECM-88, Lubniewice, 247-283, 1998,

Sawicki J., Analysis and Modeling of Electrochemical Machining of Curvilinear Rotary Surfaces, University Publisher, UTP University of Science and Technology, ISBN 978-83-61314-55-4, s.164, 2013. 